\title{
Recommandations pour une prise en charge en réseau des patients présentant une insuffisance cardiaque en Suisse
}

\author{
Task Force «Herzinsuffizienz \\ Disease Management" \\ du groupe de travail de la SSC, \\ de la SSMI et de la SSMG*

\footnotetext{
* Membres de la Task Force: Peter Buser (président) ${ }^{\mathrm{a}}$, Hans Peter Brunner-La Rocca ${ }^{a}$, Marcia Leventhal a, Romy Mahrer ${ }^{\mathrm{a}}$, Paul Mohacsia , Karl Nuescha, Pierrre Périat ${ }^{\mathrm{c}}$, Charles Seydoux ${ }^{\mathrm{a}}$, Susanne Stöhr ${ }^{\mathrm{b}}$, Hans Ueli Zürcher ${ }^{\mathrm{b}}$

b Société Suisse de Médecine interne (SSMI) générale (SSMG)
} \\ a Société Suisse de Cardiologie (SSC) \\ c Société Suisse de Médecine
}

\section{Introduction}

L'insuffisance cardiaque est définie comme un syndrome qui se présente cliniquement par des symptômes (dyspnée, intolérance à l'effort, œdèmes des membres inférieurs, etc.), des critères objectifs évaluant la fonction cardiaque (diminution de la fonction systolique ou diastolique du ventricule gauche), et une réponse positive à un traitement spécifique [1]. Les causes les plus fréquentes de cette atteinte dans les pays industrialisés sont la maladie coronaire et l'hypertension artérielle. Des étiologies plus rares peuvent être une atteinte primaire du myocarde (cardiomyopathie), des valvulopathies ou des maladies congénitales. En raison de l'évolution démographique de ces pays, en particulier par l'augmentation de la durée de vie et l'amélioration de la survie par la prise en charge de maladies cardiaques potentiellement fatales, on relève une augmentation de la prévalence de l'insuffisance cardiaque au cours de ces dernières années. Cette prévalence va encore augmenter au cours des $10-15$ prochaines années, de telle sorte que l'on peut parler d'épidémie d'insuffisance cardiaque pour les prochaines décennies [1]. Malgré les améliorations importantes dans la prise en charge de l'insuffisance cardiaque par l'administration de traitements médicamenteux et interventionnels, la mortalité de cette affection est de 30 à $40 \%$ au cours des 5 années suivant le diagnostic. Cette mortalité reste trop élevée, la qualité de vie est souvent très réduite et les coûts des hospitalisations sont importants [2]. Chez les patients de plus de 65 ans qui sont hospitalisés pour une décompensation cardiaque, le taux de ré-hospitalisation au cours des 6 mois suivants est de l'ordre de 15 à 50\% selon le diagnostic de la maladie cardiaque de base et les comorbidités associées [3]. Pour cette raison, l'insuffisance cardiaque est dans ce groupe d'âge la cause la plus fréquente d'hospitalisation. Les raisons principales d'une décompensation aiguë sont la compliance médicamenteuse diminuée dans le cadre d'un traitement complexe, la difficulté de suivre des mesures diététiques conseillées ainsi que l'insuffisance des mesures d'auto- contrôle à domicile, l'absence de réaction rapide et efficace face à une aggravation clinique, le manque de compréhension de la pathologie et les possibilités d'optimalisation thérapeutique [4].

$\mathrm{Au}$ cours des dernières années, de nombreuses études ont démontré que le développement d'un programme de prise en charge en réseau de l'insuffisance cardiaque peut réduire la mortalité des patients concernés s'il est associé à un traitement médicamenteux optimal. Ces programmes permettent également de réduire la morbidité, le taux de ré-hospitalisation et d'améliorer la qualité de vie [5-9]. Les points importants de développement d'un tel programme consistent en la prise en charge du patient en insuffisance cardiaque par un groupe médical multidisciplinaire, l'organisation précoce et globale de la sortie de l'hôpital, des contrôles ambulatoires fréquents, la reconnaissance précoce de signes d'aggravation de la maladie cardiaque, l'optimalisation du traitement médicamenteux grâce aux recommandations publiées, de même que l'information et le conseil donnés au patient. L'intégration des ces aspects dans la prise en charge de l'insuffisance cardiaque a aussi été acceptée dans les directives pour le diagnostic et la prise en charge de l'insuffisance cardiaque chronique de la Société Européenne de Cardiologie et de l'American Heart Association [1, 10]. Quoi qu'il en soit, de nombreuses questions restent ouvertes touchant plus particulièrement l'équipe d'intervention, le rapport coût/efficacité d'un tel programme, et le choix des patients qui devraient le plus profiter de cette prise en charge. D'autre part, la majorité des études était dirigée par des centres hospitaliers et il existe peu de données de la pratique ambulatoire.

Il est difficile de comparer le système d'organisation de la santé publique dans les différents pays européens ou aux Etats-Unis. Les résultats des études qui ont été effectuées aux USA, en Angleterre ou dans le Benelux ne peuvent pas nécessairement être appliqués à la Suisse, en particulier dans le contexte compliqué des réseaux de soins [17]. La Suisse possède un réseau assez

buser@email.ch 
dense et bien organisé de la prise en charge dans le domaine de la santé, de sorte qu'un programme centralisé de la prise en charge du patient en insuffisance cardiaque devrait conduire à un certain succès. Comme les discussions politiques concernant la santé en Suisse intègrent de plus en plus la notion de la prise en charge globale, ce que le présent réseau essaie de mettre sur pied, les recommandations proposées ont comme but de formuler les considérations minimales pour mettre sur pied un tel programme. Dans ce contexte, seront intégrés les évidences cliniques déjà publiées de même que seront analysées les considérations spécifiques de l'organisation de la santé suisse.

\section{Buts d'un programme de prise en charge de I'insuffisance cardiaque en réseau}

Les buts d'un tel programme en réseau sont l'amélioration de l'état clinique et du pronostic des patients présentant une insuffisance cardiaque chronique, tout en réduisant les coûts de cette prise en charge. Pour ce faire, 4 objectifs principaux ont été déterminés:

1. réduction du nombre d'hospitalisation et de ré-hospitalisation et réduction du temps d'hospitalisation;

2. amélioration de la qualité de vie et du bien être du patient;

3. amélioration du pronostic;

4. réduction des coûts en considérant le nombre d'hospitalisations.

\section{Collectif de patients}

Ce réseau de soins doit principalement toucher l'ensemble des patients chez lesquels le diagnostic d'insuffisance cardiaque a été posé. L'importance de la prise en charge ne doit cependant pas suivre un schéma fixe mais doit être adapté à la situation spécifique de chaque patient.

La meilleure évidence (niveau d'évidence A, recommandation I) de l'efficacité de cette prise en charge à ce jour a été reconnue pour les pa- tients présentant une insuffisance cardiaque progressive, particulièrement après une décompensation récente $[5-9,11]$. Ces patients profitent grandement d'un réseau de soins de ce type qui permet un suivi initial intensif, répété au cours des mois ultérieurs, voire plus longtemps. Chez les patients stables, le niveau d'évidence est moins grand. Les données récentes démontrent cependant que ces patients pourraient tout de même bénéficier d'une prise en charge en réseau de ce type [12,13]. La fréquence et la répétition de la prise en charge sont cependant moins claires. Quoi qu'il en soit, des informations dirigées sur les aspects mentionnés ci-dessous gardent tout leur sens chez ces patients également. Il est ainsi justifié de recruter dans un tel réseau de soin des patients qui présentent un haut risque de décompensation (évidence $B$, recommandation IIa). Finalement, il y a des groupes de patients qui ne vont que peu bénéficier des lignes directrices générales d'un tel réseau, par exemple les patients âgés ou présentant une démence. Chez ces patients, il s'agirait plutôt d'incorporer la parenté dans le réseau de soins.

L'indication à une telle prise en charge en réseau est d'ordre médical. Elle est posée par le médecin hospitalier responsable du patient quand celui-ci a été hospitalisé pour une décompensation cardiaque. Pour les patients ambulatoires, cette indication est posée par le médecin traitant (cf. «Réseau»).

\section{Prise en charge en réseau pendant I'hospitalisation}

Une insuffisance cardiaque est souvent diagnostiquée pour la première fois dans le cadre d'une hospitalisation. La règle de base est d'offrir la possibilité d'une prise en charge par un réseau de soins à tous les patients qui sont hospitalisés pour une décompensation d'une insuffisance cardiaque, ou chez qui on met en évidence une insuffisance cardiaque symptomatique à cette occasion. La nécessité d'une prise en charge spé-

Tableau 1

Définition des classes de recommandations et du niveau d'évidence clinique.

\section{Classes de recommandations}

Classe I Avis général que la recommandation est utile

Classe Ila Recommandation controversée, mais plutôt utile

Classe Ilb Recommandation controversée, mais utilité moins bien établie

Classe III Avis général que l'utilité de la recommandation n'est pas

définie, mais pourrait dommageable ou dangereuse
Niveau d'évidence

Grade A Plusieurs grandes études randomisées

Grade B Une seule grande étude randomisée ou plusieurs grandes études non randomisées

Grade C Avis d'experts et/ou plusieurs études plus petites 
cifique de l'insuffisance cardiaque en milieu hospitalier a déjà été documentée en Suisse dans des études antérieures [14]. Les buts principaux d'une prise en charge en réseau pendant l'hospitalisation, et jusqu'à la sortie d'hôpital, sont d'informer le patient de son affection médicale, d'initier les conseils généraux portant sur l'insuffisance cardiaque, d'optimaliser le traitement médical et finalement d'organiser de façon globale le retour à domicile (recommandation I, niveau d'évidence A) [5-9, 11].

L'information initiale au patient est la tâche du médecin responsable, mais devrait également intégrer un cardiologue ou un spécialiste dans la prise en charge de ces patients. Au cours de la première discussion, le médecin responsable devrait informer le patient de son diagnostic et des causes de sa décompensation cardiaque. Ces éléments devraient être expliqués au patient qui devrait également recevoir un plan de la prise en charge (incluant l'importance du conseil spécifique dans le cadre de l'insuffisance cardiaque). Le patient devrait également recevoir la documentation mise à disposition par la Fondation Suisse de Cardiologie en lui demandant d'en prendre connaissance jusqu'à la première discussion

Figure 1

Les cercles du programme de prise en charge des patients avec insuffisance cardiaque en réseau.

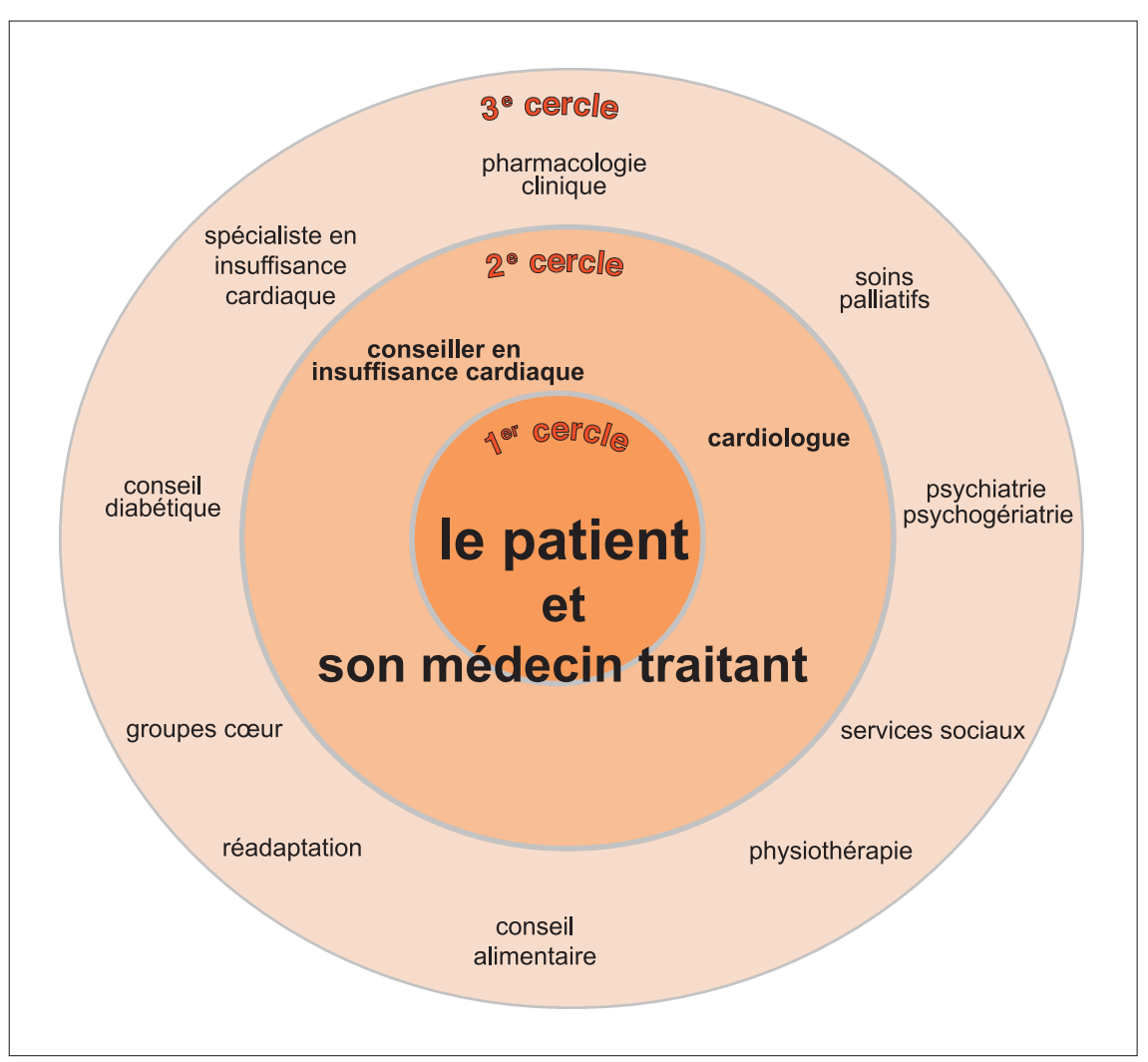

spécifique à ce sujet. D'autres programmes interactifs peuvent également être proposés au patient, comme par exemple, sur le site www. heartfailure.ch.

\section{Conseils généraux concernant l'insuffisance cardiaque}

Le médecin en charge de cette discussion spécifique devrait, au cours de cette première visite (visite 1) prendre contact avec le patient et laisser à celui-ci la possibilité de poser des questions concernant la documentation mise à disposition (env. $20 \mathrm{~min}$ ). Au cours d'une deuxième visite (visite 2, env. 60 min), devraient être abordée de façon détaillée les éléments principaux de cette information au patient, comme par exemple la compliance médicamenteuse, l'aide à la prise médicamenteuse (par l'intermédiaire d'un semainier par exemple), l'aggravation des symptômes de l'insuffisance cardiaque, les facteurs de risque, les contrôles quotidiens, en particulier le poids, l'apport en sel et en liquide. De même devrait être abordée l'importance de l'activité physique après la sortie de l'hôpital. La préparation de la sortie devrait être abordée au cours d'une troisième visite (visite 3, env. 20 min). Le médecin responsable du patient devrait aborder l'ensemble des conseils donnés dans la documentation mise à disposition ainsi que les éléments de la lettre de sortie. La documentation concernant ces conseils sera annexée à la lettre de sortie de sorte que la personne responsable du patient et les conseillers ultérieurs puissent l'utiliser en cas de besoin après la sortie de l’hôpital.

\section{Organisation du retour à domicile}

La sortie de l'hôpital et les contrôles ultérieurs sont des éléments à planifier suffisamment à l'avance entre le médecin en charge du patient, le cardiologue et le réseau de soins ambulatoires. Le médecin responsable du patient à l'hôpital prend contact avec le collègue ambulatoire par téléphone. A cette occasion, seront abordés l'évolution au cours de l'hospitalisation, le traitement et le moment opportun du premier rendez-vous ambulatoire. Il s'agira également d'informer ce médecin de l'organisation du réseau de soins et de l'importance de la poursuite de ces conseils spécifiques qui devraient être dispensés par un cardiologue si le médecin traitant ne l'est pas. Le premier rendez-vous auprès du réseau de soins ambulatoires devrait alors être déterminé. Celui-ci devrait optimalement se faire dans un délai de deux semaines après la sortie de l'hôpital, puis à un mois, deux mois, six mois et douze mois [5-9]. 


\section{Réseau de soins}

Le programme de réseau de soins de l'insuffisance cardiaque est un programme de prise en charge et d'éducation qui permet de soutenir la relation médecin-patient, ainsi que de représenter une possibilité structurée et simplifiée de discussion pour des patients présentant une insuffisance cardiaque et posant un problème spécifique ou urgent. Ce système englobe le médecin traitant, le conseiller en insuffisance cardiaque, le cardiologue, des spécialistes médicaux de l'insuffisance cardiaque, des programmes de réadaptation cardiaque, des conseils diététiques, diabétiques, des «groupes cœurs», les physiothérapeutes, l'administration sociale de la région, la prise en charge psychologique ou psychiatrique et les structures de soins palliatifs [1]. Afin de rendre compte de ce travail en réseau de façon plus visuelle, et de prendre en considération les éléments existants déjà en Suisse, l'organisation a été caractérisée en trois cercles (fig. 1).

\section{Premier cercle: le patient et le médecin traitant}

$\mathrm{Au}$ centre de ce réseau se trouve le patient. Le médecin traitant et le patient forment un partenariat actif dont le but est d'optimaliser l'état clinique du patient par une amélioration ou une stabilisation de son insuffisance cardiaque.

\section{Le patient avec insuffisance cardiaque}

Comme partenaire actif dans ce réseau, le patient doit être capable de gérer son traitement (prise médicamenteuse, comportement), de reconnaître les signes et les symptômes d'une aggravation de l'insuffisance cardiaque, de les surveiller, de les interpréter et d'agir en conséquence. Il contrôle et documente régulièrement son poids et sa pression artérielle si cela est notifié.

\section{Le médecin traitant}

On considère comme médecin traitant celui qui a le contact médical le plus proche du patient. Celui-ci peut très bien être un généraliste ou un autre spécialiste. Les recommandations pour la prise en charge de l'insuffisance cardiaque se basent sur les dernières prescriptions de la Société Suisse de Cardiologie (SSC; en préparation, publication fin 2006), de la Société Européenne de Cardiologie (SEC) [1] et de l'American Heart Association (AHA) [10]. Ce médecin réfère le patient au réseau de soins spécifique est reste en contact avec les personnes actives dans ce programme.

\section{Deuxième cercle: conseiller en insuffisance cardiaque et cardiologue}

Conseiller en insuffisance cardiaque (CIC) Le CIC est une personne dans le domaine des soins qui a bénéficié d'une formation spécifique reconnue par le groupe de travail «Insuffisance cardiaque» de la Société Suisse de Cardiologie pour la prise en charge des patients présentant une insuffisance cardiaque. Le CIC est responsable de l'éducation du patient et le conseille quant aux prescriptions médicales. Il soutient le patient afin d'améliorer sa compliance, l'aider à reconnaître précocement l'aggravation des symptômes ou des signes clinique et est capable d'agir en conséquence, comme par exemple de référer le patient plus rapidement que prévu à son médecin traitant.

\section{Le cardiologue spécialiste}

Ce cardiologue est le partenaire de l'ensemble des personnes dispensant les soins, du médecin hospitalier responsable du patient et du CIC pour des questions en relation avec le diagnostic et le traitement de l'insuffisance cardiaque.

\section{Troisième cercle: prise en charge élargie}

\section{Spécialiste en insuffisance cardiaque}

Les spécialistes dans le domaine de la prise en charge de l'insuffisance cardiaque, sont des spécialistes cardiologues ayant une formation spécifique et une expérience dans la prise en charge des patients présentant des problèmes complexes liés à cette pathologie. La formation continue peut se faire dans un centre qui propose un programme de prise en charge de ces patients spécifiques. Ils peuvent être appelés à donner des conseils sur la prise en charge de patients présentant une situation particulièrement difficile. Ils sont également les partenaires directs pour la discussion des thérapies adjuvantes comme le pacing biventriculaire, la mise en place d'assistance circulatoire ou l'organisation d'une transplantation cardiaque.

\section{Programme de réadaptation cardiaque et «groupe cour»}

Dans le cadre des programmes de réadaptation cardiaque et des «groupes cœurs» se retrouvent des patients présentant une cardiopathie, et qui sont sous la direction d'un médecin responsable du programme ou d'une personne formée dans ce domaine (comme par exemple les physiothé- 
rapeutes dans les «groupes cœurs»). Le but est d'améliorer la tolérance à l'effort de ces patients, de même que d'échanger des expériences avec les autres personnes impliquées dans le suivi de ceux-ci. Les patients présentant une insuffisance cardiaque pourraient être intégrés dans ces groupes qui sont organisés à un niveau régional, et soutenus par la Fondation Suisse de Cardiologie.

\section{Conseiller diététique}

Le conseiller diététique peut apporter des informations et une éducation dans le cadre de l'alimentation des patients, en particulier concernant le régime sans sel et la restriction hydrique. Ce conseiller joue également un rôle important dans les conseils aux patients présentant des problèmes liés à l'excès pondéral ou à la cachexie, représentant dans ce contexte un risque de morbidité-mortalité plus élevé.

\section{Autres services}

Conseiller diabétique, physiothérapie, services sociaux, prise en charge psychologique et psychiatrique, pharmacologue clinique, soins palliatifs, prise en charge des fins de vie. L'ensemble de ces services reste à disposition selon la nécessité.

\section{Communications et contacts}

La communication à l'intérieur du réseau est nécessitée par le besoin d'une prise en charge cohérente et harmonieuse des patients.

\section{Moyens de communication}

\section{A la sortie de l'hôpital}

Le jour de la sortie d'hôpital, le médecin traitant ambulatoire et le CIC seront informés, par l'intermédiaire d'un rapport de sortie, par le médecin responsable du patient à l'hôpital sur les points spécifiques suivants:

1. diagnostic de l'insuffisance cardiaque et diagnostics secondaires importants;

2. poids d'entrée, poids de sortie, objectif du poids optimal visé;

3. médication actuelle et indications sur les possibilités de thérapie maximale;

4. date de sortie;

5. situation actuelle du conseil en insuffisance cardiaque et détermination des objectifs ultérieurs;

6. avis de réception par le médecin traitant et le CIC.

\section{Prise en charge ambulatoire}

Le succès d'un tel programme dépend de l'information réciproque régulière de l'évolution clinique.

\section{Médecin traitant}

Il représente le pouvoir décisionnel central. Il est directement responsable de la prise en charge du patient, de son traitement et de ses modifications, au besoin après discussion avec le cardiologue ou/et le CIC. Il reste en contact avec le CIC et lui réfère le patient pour des conseils complémentaires. Pour des questions spécifiques et des problèmes qu'il rencontrerait, il pourrait se référer aux cardiologues impliqués.

\section{Conseiller en insuffisance cardiaque}

Le CIC oriente le médecin traitant sur l'évolution du patient et les buts atteints ou non. Il peut faire des propositions au médecin traitant concernant des améliorations du traitement, ou la nécessité de demander l'avis aux cardiologues impliqués ou aux spécialistes désignés en insuffisance cardiaque. Le médecin traitant décide alors des dispositions à prendre.

\section{Le spécialiste de cardiologie}

il reste à disposition du CIC et ainsi qu'à celle des médecins concernés. Il peut faire des propositions d'amélioration de traitement au médecin traitant et éventuellement des propositions d'investigations complémentaires. Il soutient le CIC dans sa tâche.

\section{Information aux patients}

La première évaluation par le CIC après la sortie de l'hôpital devrait, selon les directives de la Société Européenne de Cardiologie, se faire dans les dix jours qui suivent cette sortie. Cette première séance d'information et de conseil peut également s'adresser à la parenté sur leur demande.

\section{Contenu du programme d'information et de conseil}

Ce contenu peut être ordonné de la façon suivante (recommandation I, évidence A) [1, 15, 16]:

\section{Compréhension de la pathologie}

Une meilleure compréhension des symptômes de l'insuffisance cardiaque et des éléments pouvant apparaître dans l'évolution de cette pathologie, permet au patient de participer directement à son traitement. Afin d'éviter des réticences ou des mauvaises compréhensions des 
traitements proposés, et de bien thématiser la présentation de la maladie de façon individuelle, il est nécessaire d'établir un plan thérapeutique avec le patient.

\section{Autocontrôles}

Les patients doivent apprendre à s'observer, à prendre en considération les modifications des symptômes afin de transmettre les informations au CIC ou au médecin traitant. Idéalement, il devrait apprendre à interpréter les symptômes d'une aggravation (par exemple ceux de la rétention hydrosaline) et de réagir rapidement par l'adaptation de son traitement dans les limites des indications du médecin traitant. Les patients contrôlent et documentent régulièrement leur poids et le cas échéant, la tension artérielle.

Diète, effets toxiques et facteurs de risques On conseillera au patient de suivre une diète pauvre en sel et de réduire leur rapport hydrique pour limiter la rétention hydrosaline. En même temps, l'alimentation générale devra être dirigée pour réduire le risque d'aggravation de l'insuffisance cardiaque. Comme celle-ci pourrait être la conséquence d'un abus d'alcool, une abstinence rigoureuse devrait être obtenue. On devrait également proposer un programme «stop tabac».

\section{Exercices physiques}

Le patient devrait, avec les conseils du CIC, développer un plan d'exercices physiques individuel.

\section{Médicaments}

L'importance de la prise régulière et précise des médicaments doit être abordée. Celle-ci peutêtre optimalisée par des aides à la prise médicamenteuse ou sous forme de systèmes d'alarme (par exemple avec des montres-réveils annonçant l'heure de la prise de médicaments) et le patient devrait être sensibilisé à leur utilisation.

\section{Scénario d'urgence}

Des numéros d'urgence et des heures de disponibilité du CIC ou du médecin traitant, de même que des attitudes concrètes en cas de rapide détérioration de la situation devraient être transmis de façon claire.

\section{Documentation personnelle}

L'évolution de la maladie nécessite une documentation régulière du poids et des symptômes, de même que de la tension artérielle et du pouls dans certaines situations précises. Dans ce but, le patient est instruit à utiliser un journal quotidien qui devrait lui être mis à disposition.

\section{Problème psychosociaux}

L'état dépressif est fréquent chez les patients présentant une insuffisance cardiaque. Ceci doit donc être reconnu et abordé par le patient et la parenté, de même que devraient être mis à disposition les moyens pour remédier à une situation d'urgence dans ce domaine.

\section{Etapes de l'éducation du patient}

Trois étapes sont possibles afin de limiter la complexité et de rendre compréhensible la transmission des connaissances (recommandation I, niveau d'évidence C). Ces étapes devraient être suivies par une supervision de la connaissance du patient et de l'évaluation des capacités d'indépendance de ce dernier. Une étape ne devrait être suivie d'une autre que si tous les problèmes en relation avec son activité quotidienne ont été abordés. Ceci va dépendre des capacités d'apprentissage et de la volonté du patient et de ses proches. L'évaluation de ses capacités, des objectifs atteints par l'éducation dans une étape et l'ensemble des conseils devraient être effectués par le médecin traitant et rapportés au CIC.

Le but retenu du niveau I est de diminuer le risque de ré-hospitalisation en sensibilisant le patient sur l'évolution de sa maladie. Les connaissances et les buts des niveaux II et III devraient permettre de développer les capacités d'indépendance du patient, afin d'évaluer de façon personnelle l'évolution prévisible de son insuffisance cardiaque. L'évaluation des connaissances et des objectifs fixés sera effectuée par un questionnaire standardisé à la fin de l'éducation.

\section{Prise en charge à long terme / nombre de contacts}

\section{Durée initiale du programme de prise en charge de l'insuffisance cardiaque}

Tout patient hospitalisé en raison d'une insuffisance cardiaque devrait faire partie d'un système d'éducation en réseau de ce type. Le programme dure aussi longtemps que le patient n'a pas atteint les différentes étapes successives. Le but minimal devrait être d'obtenir les connaissances de la première étape et d'avoir rempli les conditions requises à ce niveau.

\section{Apprentissage}

Les répétitions régulières permettent de maintenir un niveau de connaissances et de l'intégrer 
dans son activité quotidienne. Il est ainsi nécessaire de rafraîchir ces connaissances après la fin de l'éducation initiale, à des intervalles de 6 à 12 mois. La comparaison des durés de ces types de cours dans le cadre de programmes standardisés (court: 0-3 mois, moyen: 3-6 mois, long: plus de 6 mois) semble démontrer que cette forme de répétition permet d'obtenir de meilleurs résultats sur la prise en charge générale, comme le montre une méta-analyse [5]. L'indication à la répétition de ces cours se fera après discussion entre le médecin traitant et le CIC. Des évaluations régulières par des questionnaires standardisés permettent d'assurer un contrôle de qualité.

\section{Contacts personnels}

Différentes études semblent montrer que, dans le cadre de la prise en charge des patients avec insuffisance cardiaque, les contacts personnels directs ou par téléphone diminuent de façon significative la mortalité et le taux de ré-hospitalisation $[6,8]$. Il semble donc que différents types de contrôles puissent être organisés dans le cadre de la prise en charge multidisciplinaire par le médecin traitant, le cardiologue et le CIC (par des visites ou des contacts téléphoniques).

\section{Assurance de qualité et contrôle de qualité}

Les évaluations et les contrôles réguliers de la qualité de la formation sont nécessaires pour assurer le succès d'une prise en charge en réseau. D’une part, il est nécessaire d'évaluer régulièrement si le patient a compris la notion de la prise en charge en réseau et qu'il a adapté son comportement à ces informations. D'autre part, tous les collaborateurs participant à ce genre de réseau doivent remplir un certain nombre de conditions de qualité, qui devrait également être évaluées régulièrement.

\section{Evaluation des patients}

L'évaluation des patients repose d'une part sur l'acquisition et l'état des connaissances et sur leur attitude, et d'autre part sur la qualité de vie, les plaintes et l'état clinique. Dans ce but, il sera mis en place un formulaire standardisé qui évalue ces différents aspects par un petit nombre de questions. Ces formulaires doivent aussi permettre d'évaluer l'état clinique et d'en déterminer sa stabilité. De plus, il sera déterminé si l'ensemble des informations pourra être distribué de façon efficace à une échelle plus large après la fin de la formation et des conseils donnés aux patients. Il est ainsi important de ne pas évaluer seulement les connaissances acquises, mais surtout le com- portement face à la maladie. Cette évaluation offre une base permettant de déterminer d'autres besoins du système de prise en charge par réseau. Ainsi l'on pourra par exemple évaluer si certains patients devraient bénéficier de conseils plus approfondis, ou nécessitent à plus long terme un rafraîchissement des connaissances. Cette évaluation est également nécessaire pour déterminer si les buts fixés pour le réseau de soins ont été atteints. Finalement, elle devrait permettre de s'adapter aux résultats des différentes études cliniques dans ce domaine.

Le formulaire standardisé devrait en particulier évaluer les aspects suivants:

- état clinique du patient;

- capacités du patient;

- compliance du patient.

Il est utile que le patient porte sur lui les informations importantes concernant sa pathologie. Dans ce but, il sera élaboré un passeport insuffisance cardiaque qui devrait contenir ces éléments mais également l'objectivation des évaluations régulières des patients.

Prestataires de soins impliqués dans la prise en charge en réseau de l'insuffisance cardiaque

L'ensemble des collaborateurs d'un tel réseau doit remplir des connaissances minimales précises. Celles-ci sont déterminées dans les propositions élaborées par le groupe de travail insuffisance cardiaque de la Société Suisse de Cardiologie, en collaboration avec la Société Suisse de Médecine Interne et la Société Suisse de Médecine Générale. Les personnes proposant ce programme structuré qui contient ces exigences, doivent avoir obtenu une reconnaissance spécifique du groupe de travail de la Société Suisse de Cardiologie. Ce groupe de travail organise également la formation des CIC et permet l'obtention d'un diplôme en accord avec ses statuts de formation.

\section{Eléments de base nécessaires à la mise en place d'un programme de prise en charge d'un réseau de l'insuffisance cardiaque en Suisse}

\section{Buts}

- Réduire le nombre et la durée des hospitalisations et des ré-hospitalisations;

- amélioration de la qualité de vie et du bienêtre du patient;

- amélioration du pronostic;

- réduction des coûts occasionnés par les hospitalisations. 


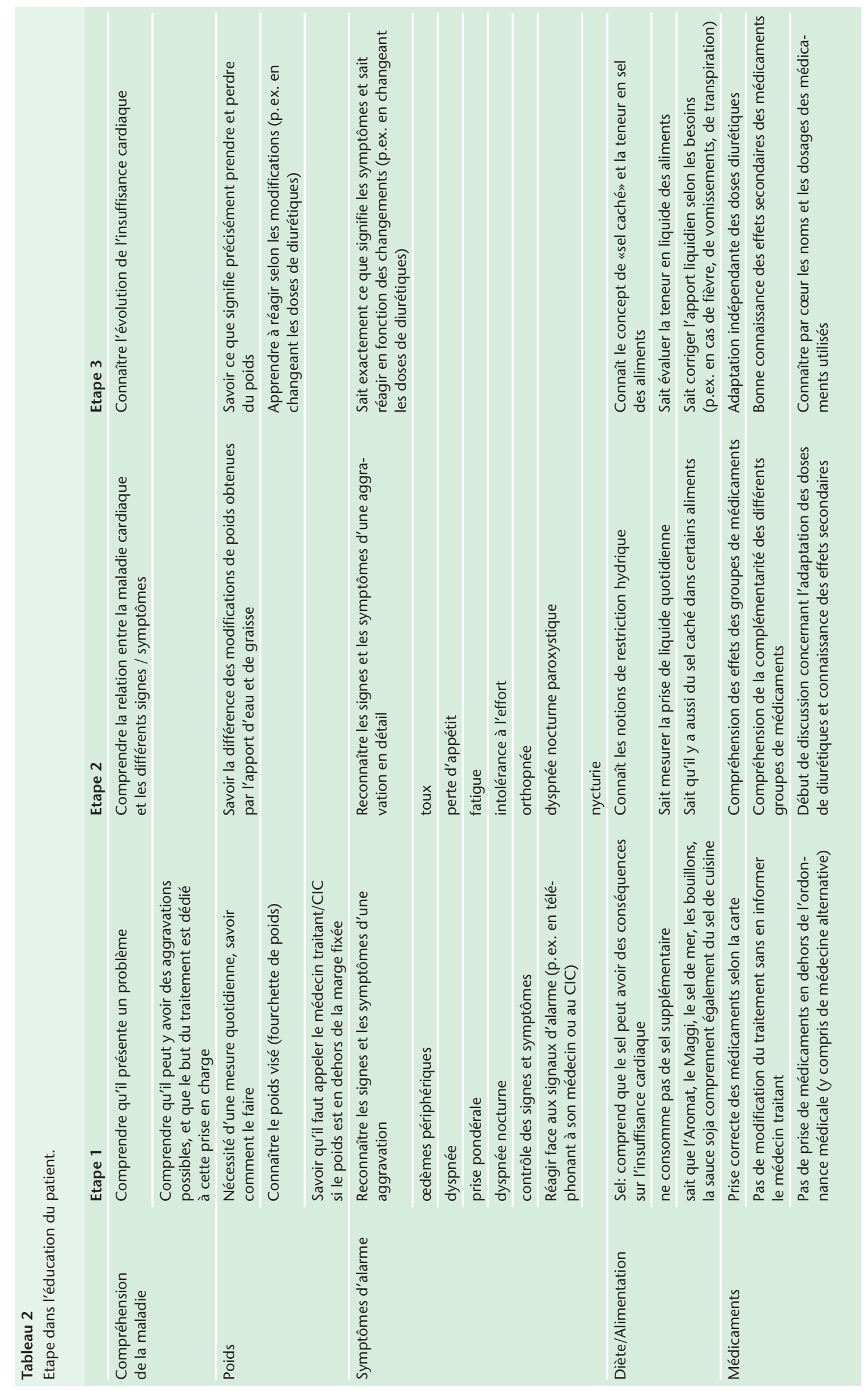



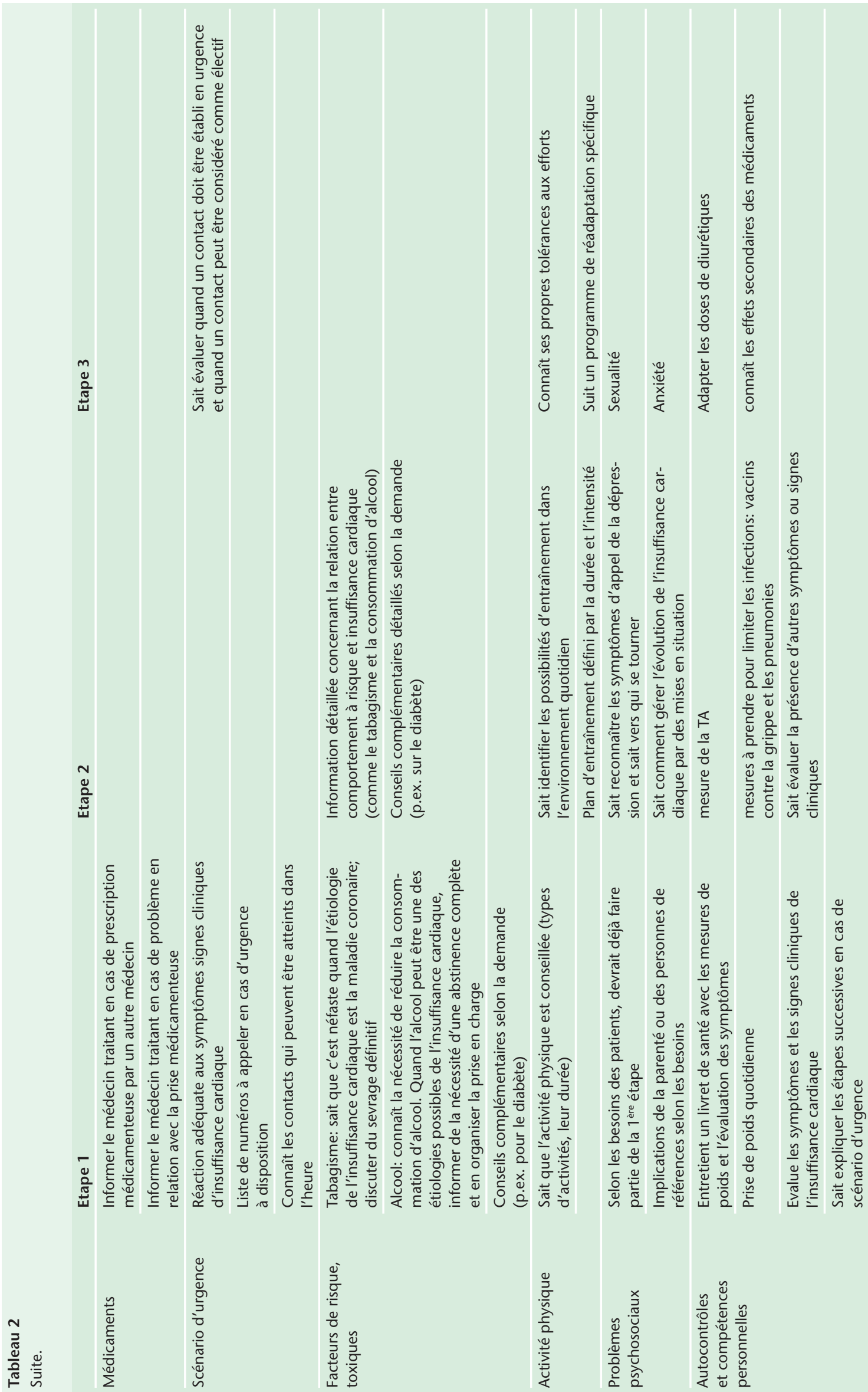


\section{Sélection du patient et détermination de l'indication}

- Tout patient présentant une insuffisance cardiaque, en particulier tout ceux qui ont été hospitalisés pour une décompensation;

- l'indication à la prise en charge est déterminée à l'hôpital par le médecin responsable du patient;

- en milieu ambulatoire, l'indication est posée par le médecin traitant du patient.

\section{Prise en charge des patients en insuffi-} sance cardiaque à l'hôpital

- Concerne tous les patients ayant une insuffisance cardiaque;

- information au patient par le médecin traitant, le cardiologue et les spécialistes en insuffisance cardiaque;

- début des conseils en insuffisance cardiaque par un conseiller spécifique;

- optimalisation du traitement de l'insuffisance cardiaque;

- planification de la sortie de l’hôpital.

\section{Organisation du programme de prise en} charge de l'insuffisance cardiaque en réseau

- Le patient, qui est au centre de la prise en charge;

- le médecin traitant (respectivement le généraliste, le cardiologue);

- le conseiller en insuffisance cardiaque (CIC, le soignant avec une formation en insuffisance cardiaque);

- le cardiologue spécialiste, le spécialiste en insuffisance cardiaque;

- le programme de réhabilitation cardiaque, les «groupes cœurs»;

- les conseillers diététiques;

- les services complémentaires selon la nécessité: conseiller diabétiques, physiothérapeutes, service social, la prise en charge psychologique et psychiatrique, la pharmacologie clinique, les soins palliatifs.

\section{Communication}

- A l'hôpital: le patient avec le médecin traitant spécialiste et le CIC;

- à la sortie de l'hôpital: le médecin responsable du patient, le médecin traitant ambulatoire et le CIC;

- premier cercle: le patient avec le médecin traitant;

- deuxième cercle: le patient et le médecin traitant avec le cardiologue et le CIC;

- troisième cercle: les personnes impliquées dans les deux premiers cercles, respectivement le patient et le médecin traitant, en associa- tion avec les spécialistes et les services autres prestataires de soins nécessaires.

\section{Education du patient}

- Programme structuré portant sur la compréhension de la pathologie, les contrôles à domicile, la documentation, la diète, les effets secondaires et les effets toxiques des médicaments, les facteurs de risques, l'entraînement physique, les médicaments, le scénario en cas d'urgence, les problèmes psychosociaux;

- adaptation individuelle des étapes d'apprentissage et des exigences du programme;

- le but est d'atteindre les objectifs de la première étape d'éducation pour tous les patients.

\section{Prise en charge à long terme}

- Evaluation des connaissances du patient de façon répétée par l'intermédiaire d'un questionnaire standardisé avec le médecin traitant;

- contact téléphonique pour évaluer la connaissance des patients avec un questionnaire standardisé, par le médecin traitant ou le CIC;

- possibilité d'offrir des cours afin de répéter l'ensemble des informations.

\section{Contrôle de qualité et assurance de la qualité}

- Evaluation du patient avec un questionnaire standardisé portant sur son état de santé, ses capacités physiques et intellectuelles, et sa compliance médicamenteuse;

- évaluation du réseau concernant le maintien des objectifs;

- reconnaissance du réseau par la Société Suisse de Cardiologie.

\section{Références}

1 Swedberg K, Cleland J, Dargie H, et al. Guidelines for the diagnosis and treatment of chronic heart failure: executive summary (update 2005): The Task Force for the Diagnosis and Treatment of Chronic Heart Failure of the European Society of Cardiology. Eur Heart J. 2005;26:1115-40.

2 Krum H, Gilbert RE. Demographics and concomitant disorders in heart failure. Lancet. 2003; 362:147-58.

3 Lenzen MJ, Scholte op Reimer WJ, Boersma E, et al. Differences between patients with a preserved and a depressed left ventricular function: a report from the EuroHeart Failure Survey. Eur Heart J. 2004;25:1214-20.

4 Nieminen MS, Bohm M, Cowie MR, et al. Executive summary of the guidelines on the diagnosis and treatment of acute heart failure: the Task Force on Acute Heart Failure of the European Society of Cardiology. Eur Heart J. 2005;26:384-416. 
5 Roccaforte R, Demers C, Baldassarre F, et al. Effectiveness of comprehensive disease management programmes in improving clinical outcomes in heart failure patients. A meta-analysis. Eur J Heart Fail. 2005;7:1133-44.

6 Phillips CO, Wright SM, Kern DE, et al. Comprehensive discharge planning with postdischarge support for older patients with congestive heart failure: a meta-analysis. JAMA. 2004;291:1358-67.

7 Gonseth J, Guallar-Castillon P, Banegas JR, et al. The effectiveness of disease management programmes in reducing hospital re-admission in older patients with heart failure: a systematic review and meta-analysis of published reports. Eur Heart J. 2004;25:1570-95.

8 McAlister FA, Stewart S, Ferrua S, et al. Multidisciplinary strategies for the management of heart failure patients at high risk for admission: a systematic review of randomized trials. J Am Coll Cardiol. 2004;44:810-9

9 Holland R, Battersby J, Harvey I, et al. Systematic review of multidisciplinary interventions in heart failure. Heart. 2005;91:899-906.

10 Hunt SA, Abraham WT, Chin MH, et al. ACC/AHA 2005 Guideline Update for the Diagnosis and Management of Chronic Heart Failure in the Adult: a report of the American College of Cardiology/American Heart Association Task Force on Practice Guidelines (Writing Committee to Update the 2001 Guidelines for the Evaluation and Management of Heart Failure): developed in collaboration with the American College of Chest Physicians and the International Society for Heart and Lung Transplantation: endorsed by the Heart Rhythm Society. Circulation. 2005;112:e154-e235.
11 Cleland JG, Louis AA, Rigby AS, et al. Non-invasive home telemonitoring for patients with heart failure at high risk of recurrent admission and death: the Trans-European Network-Home-Care Management System (TEN-HMS) study. J Am Coll Cardiol. 2005; 45:1654-64.

12 Randomised trial of telephone intervention in chronic heart failure: DIAL trial. BMJ. 2005; 331:425.

13 Austin J, Williams R, Ross L, et al. Randomised controlled trial of cardiac rehabilitation in elderly patients with heart failure. Eur J Heart Fail. 2005 7:411-7.

14 Wagdi P, Vuilliomenet A, Kaufmann U, et al. Ungenügende Behandlungsdisziplin, Patienteninformation und Medikamentenverschreibung als Ursachen für die Notfallhospitalisation bei chronisch herzinsuffizienten Patienten. Schweiz Med Wochenschr. 1993;123:108-12.

15 Faxon DP, Schwamm LH, Pasternak RC, et al. Improving quality of care through disease management: principles and recommendations from the American Heart Association's Expert Panel on Disease Management. Circulation. 2004;109:2651-4.

16 Grady KL, Dracup K, Kennedy G, et al. Team management of patients with heart failure: A statement for healthcare professionals from The Cardiovascular Nursing Council of the American Heart Association. Circulation. 2000;102:2443-56.

17 Mohacsi P. Vernetzte Betreuung von Herzinsuffizienzpatienten. Schweiz Ärztezeitung. 2003; 84(49):2617-22. 